\title{
QUANTIFICATION OF NONLINEAR FEATURES IN CARDIOVASCULAR SIGNALS
}

\author{
Javorka M., Tonhajzerova I., Turianikova Z., Chladekova L., Javorka K., \\ Calkovska A.
}

Department of Physiology, Jessenius Faculty of Medicine, Comenius University, Martin, Slovak Republic

\begin{abstract}
The analysis of spontaneous heart rate and blood pressure oscillations under standardized conditions is a rapid, sensitive, noninvasive and reproducible tool for the assessment of cardiovascular autonomic dysfunction. Since the heart and vessels are controlled by a nonlinear deterministic system, measures derived from nonlinear systems theory are increasingly used in cardiovascular variability analysis. New nonlinear methods with applicability to real biological signals are continuously developed to quantify new aspects of cardiovascular signals with the potential to reveal subtle changes in the cardiovascular control system. Nonlinear measures provide complementary information about qualitative features of the analyzed signal. Since the analysis of variability magnitude and signal nonlinear characteristics (complexity, recurrences, time irreversibility, etc.) can provide independent information on cardiovascular system control, we suggest that simultaneous use of both groups of measures can increase the information value and thus improve the sensitivity and reliability of the detection and monitoring of the cardiovascular system dysregulation during various pathological conditions.
\end{abstract}

Key words: heart rate variability, blood pressure variability, nonlinear dynamics, complexity

\section{CARDIOVASCULAR PARAMETERS VARIABILITY}

Cardiovascular dysregulation (not only as a consequence of autonomic neuropathy) is accompanied by the markedly increased risk of severe cardiovascular complications including fatal arrhythmia and sudden cardiac death. The diagnosis of dysregulation was usually performed by evaluation of heart rate and blood pressure changes evoked by Ewing tests, including Valsalva manoeuvre, deep breathing test, orthostatic test and isometric handgrip test. However, these tests require active patient participation and cooperation, are time consuming, difficult to standardize, and carry a potential risk of adverse effects $(1,2)$.

It was shown that provoked changes as a tool to investigate the cardiovascular control could be substituted by the analysis of spontaneous cardiovascular parameters oscillations. Heart rate and blood pressure continuously fluctuate over time (Fig. 1) and are influenced by different control mechanisms maintaining cardiovascular homeostasis. The analysis of short-term (on the time scales of seconds to minutes) spontaneous heart rate and blood pressure oscillations (heart rate variability - HRV and blood pressure variability - BPV) provides important information on the autonomic control of circulation in normal and diseased conditions (4).

The analysis of HRV and BPV under standardized conditions (supine rest, orthostasis) is a rapid, sensitive, noninvasive and reproducible tool for the assessment of cardiovascular autonomic dysfunction (5). Reduction in HRV magnitude is the earliest indicator of cardiovascular dysregulation (e.g. in diabetes mellitus) (6). Since HRV originates predominantly from oscillations in parasympathetic nervous traffic and blood vessels are predominantly under sympathetic nervous system control, beat-to-beat analysis of simultaneously obtained heart rate and blood pressure signals can provide information about both components of cardiovascular control $(7,8)$.

\footnotetext{
Address for correspondence:

Assoc.Prof.Michal Javorka,MD,PhD, Department of Physiology, Jessenius Faculty of Medicine, Comenius University Mala Hora 4, 03601 Martin, Slovak Republic, Phone/fax: +421 43 4131426; e-mail: mjavorka@jfmed.uniba.sk
} 

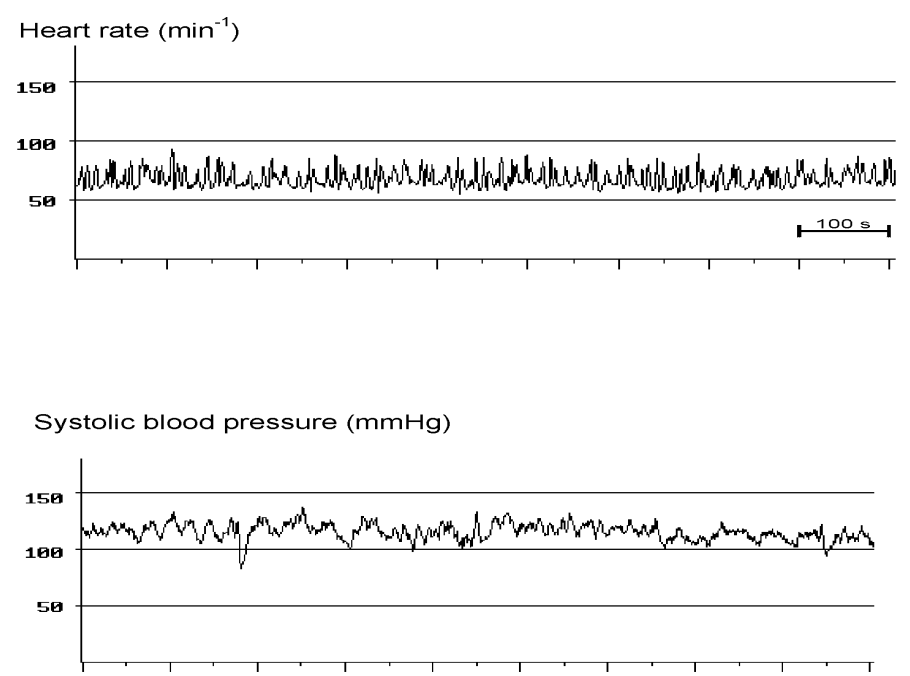

Fig. 1. Spontaneous short-term oscillations of heart rate and systolic blood pressure during supine rest. Analysis of these oscillations can provide important information on autonomic control of cardiovascular system (3)

HRV and BPV can be quantified in various ways. In 1996, the Task Force of the European Society of Cardiology and the North American Society of Pacing and Electrophysiology established a standard set of time and frequency domain measures. These numerical characteristics quantifying the magnitude of oscillations are also called linear measures because of the assumption of linear dynamics with the proposed output in the form of a set of more or less periodic oscillations (5). The linear measures are strongly mutually dependent and their values markedly overlap between healthy and pathological groups. Therefore, new parameters able to quantify additional information (independent of signal magnitude) hidden in the HRV and BPV dynamics are needed.

Importantly, HRV and BPV reflect the complex interactions of many different control loops of the cardiovascular control system. A detailed description and classification of dynamic changes using linear (time and frequency domain) measures is often insufficient. Given the complexity of the system modulating the sinus node activity, a predominantly nonlinear behavior where the output is not proportional to input has to be assumed. It was proposed that the measures from nonlinear dynamics could be important for the description of various phenomena involved in such complex control system $(9,10)$.

Since the heart and vessels are controlled by a nonlinear deterministic system, measures derived from nonlinear systems theory are increasingly used in cardiovascular variability analysis. However, the application of traditionally used nonlinear methods (developed for mathematical and physical applications mostly in 1980s-90s; e.g. correlation dimension, largest Lyapunov exponent) is limited only to long stationary signals - a condition that is only rarely met in biology (11) and their uncritical application to biological data can lead to serious pitfalls (12). New methods with applicability to real biological signals are continuously developed to quantify new aspects of short quasistationary HRV and BPV signals with the potential to reveal subtle changes in the cardiovascular control system (9).

The aim of this paper is to summarize novel nonlinear approaches developed to quantify various aspects of cardiovascular signal characteristics from short data re- 
cordings ( 5-15 min). In the following sections, the methods for assessment of complexity, recurrences in dynamics and time irreversibility are briefly introduced.

\section{CARDIOVASCULAR COMPLEXITY}

Integrated biological systems are characterized by various interacting subsystems and feedback loops processing internal and external inputs. This complex organization results in a dynamical complexity that can be revealed and quantified by the analysis of the time course of system variables under control (e.g. heart rate, systolic blood pressure). Cardiovascular regulation in the healthy human body is mediated by a variety of neural, hormonal, genetic and external interactions that operate across multiple time scales. Output variables of that system exhibit complex fluctuations - the measured output signal is characterized by great complexity. Human body is a complex adaptive system and the complexity in its behavior allows for broadest range of adaptive responses inherent to a healthy individual. A wide class of various disease states as well as aging appear to reduce this complexity hereby reducing the adaptive capacity of the individual. Therefore, the loss of complexity was proposed as a general feature of pathological dynamics (13-15).

Numerous studies have suggested that the quantification of complexity is of importance for the understanding as well as for the classification of heart rate oscillations $(14,16)$. In contrast, complexity analysis of blood pressure fluctuations have only been performed rarely (17).

\subsection{QUANTIFICATION OF COMPLEXITY}

\subsubsection{Entropy measures}

In the framework of Shannon's information theory (1946) (18), entropy is the measure of information of a given message. A message with a low entropy /information is characterized by repetition. For example, the message '123123123123'could be simply expressed by '4x123' without any loss of information. A message with high entropy/information on the other hand is characterized by little redundancy, e.g. '234365434463'.

In the case of HRV and BPV analysis, entropy measures are therefore used to quantify the complexity / regularity of heart rate / blood pressure fluctuations. Firstly, the complexity analysis of HRV was performed by calculation of Approximate Entropy $(A p E n)$ introduced by Pincus in 1991. This measure was aimed to provide widely applicable data analysis measure enabling to classify data based on their regularity (19).

Sample entropy (SampEn) is an improved version of the approximate entropy. SampEn calculates the probability that NN interval sequences (NN denotes the time duration of normal RR interval) of length $m$ that are similar within a tolerance $r$ remain similar at the next point. SampEn is precisely the negative natural logarithm of the conditional probability that a dataset of length $N$, having repeated itself within a tolerance $r$ for $m$ points, will also repeat itself for $m+1$ points, without allowing selfmatches (20) (Fig. 2). 


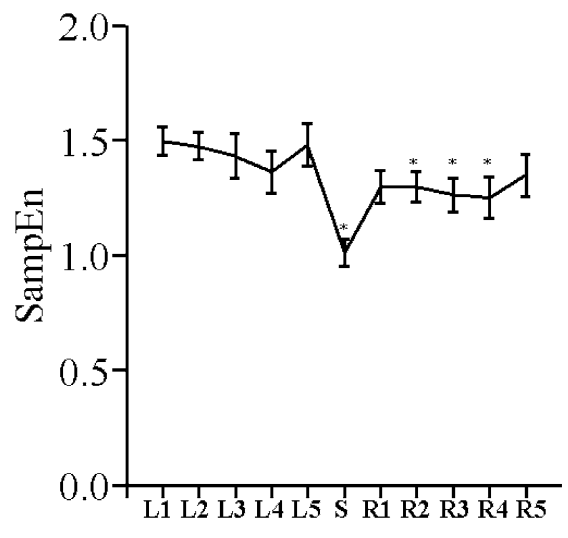

Fig. 2. Sample Entropy (SampEn) of heart rate oscillations and its changes in subject during supine rest (L1L5), orthostasis (S), and recovery after physical exercise. The reduction of SampEn during orthostasis and recovery after exercise indicates the simplification of heart rate dynamics accompanying the shift of autonomic nervous system balance towards sympathetic predominance. The asterisks indicate the significant difference from supine rest values (21)

A different way to assess entropy is based on data compression technique. In information theory, the smallest algorithm that produces a string is at the same time the entropy of that string (Chaitin-Kolmogorov entropy). Although it is theoretically impossible to develop such an algorithm, data compression techniques might provide a good approximation. The modified version of the LZ77 algorithm for lossless data compression introduced by Lempel and Ziff (1977) (22) can be used to compress time series. The ratio of the uncompressed to the compressed time series named compression entropy $\left(H_{c}\right)$ is used as a HRV and BPV complexity measure (13) (Fig. 3).

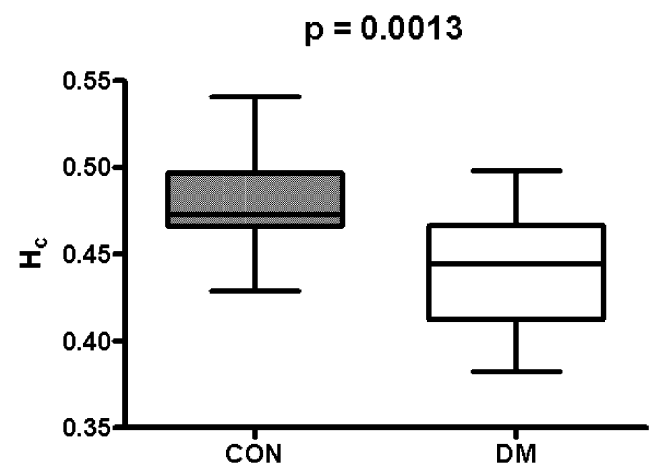

Fig. 3. Compression entropy (Hc) of heart rate is significantly lower in young patients with type 1 diabetes mellitus (DM) compared to control group (CON) according to the concept of complexity loss as an indicator of cardiac dysregulation (23) 


\subsubsection{Symbolic dynamics}

The concept of symbolic dynamics goes back to Hadammard (1898) and allows a simplified description of the dynamics of a system with a limited amount of symbols. In HRV analysis from ECG, consecutive RR intervals or their changes, respectively, are encoded according to some transformation rules into a few symbols of a certain alphabet. Subsequently, the dynamics of that symbol string are quantified, providing some more global information about the heart rate / blood pressure dynamics. Several techniques are used to describe dynamics encoded in symbolic sequence $(24,25)$.

Approach according to Voss et al (1996) (24): The difference between each NN interval and the mean NN interval is transformed into an alphabet of 4 symbols $\{0,1,2,3\}$ according to the transformation rule described in (24). Symbols '0' and'2' reflect low deviation (decrease or increase) from the mean NN interval, and symbols ' 1 ' and ' 3 ' reflect a stronger deviation (decrease or increase over a predefined limit). Subsequently, the symbol string is transformed to words of three successive symbols, e.g. '023' or '221'. The distribution of word types reflects some nonlinear properties of HRV. From this symbolic dynamics the following measures of complexity can be calculated: FORBWORD - number of word types that occur seldom, i.e. with a probability less 0.001, shannonShannon entropy over all word types as defined by Voss et al. (24). In the pathological conditions FORBWORD increases while shannon decreases reflecting the simplification of system dynamics - i.e. the loss of complexity.

Approach according to Porta et al (2001) (25): NN interval sequences are uniformly spread on six levels, resulting in 6 different symbols $\{0,1,2,3,4,5\}$. Two approaches are used for analysis of resulting symbolic time series. Normalized complexity index (NCI) is a measure of complexity and is computed as a minimum of normalized corrected conditional entropy (NCCE) as a function of $L$ (length of pattern). NCCE is a measure of the amount of information (corrected for short-term time series) carried by the $L$-th sample when the previous $L-1$ samples are known. NCCE remains constant in case of white noise; decreases to zero in case of fully predictable signals; exhibits a minimum if repetitive pattern is embedded in noise. NCI is a measure of the complexity of pattern distribution. It ranges from zero (maximum regularity) to one (maximum complexity) - the larger the NCI, the more complex and less regular the time series. The second approach is focused on the pattern classification within dynamics (26): all the patterns (symbolic sequences) with $L=3$ were grouped into 4 families according to number and types of variations from one symbol to the next one. The pattern families are: 1) patterns with no variation $(\mathrm{OV}$, all three symbols are equal); 2$)$ patterns with one variation $(1 \mathrm{~V}$, two consecutive symbols are equal and the remaining one is different); 3) patterns with two like variations ( $2 L V$, three symbols form an ascending or descending ramp), 4) patterns with two unlike variations ( $2 U V$, three symbols form a peak or a valley). The rates of occurrence of these patterns are indicated as $O V \%, 1 \mathrm{~V} \%, 2 L V \%$ and $2 U V \%$ (Fig. 4). 
A

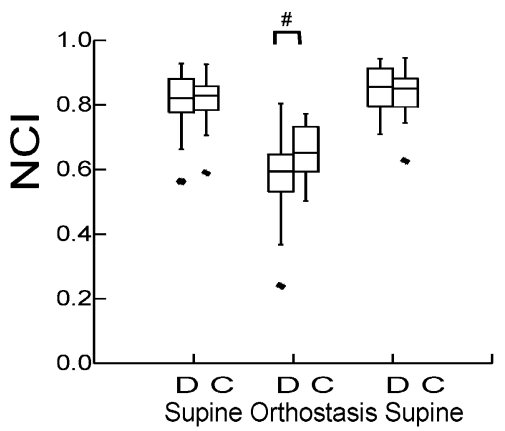

Fig. 4. Symbolic dynamics analysis of heart rate during orthostasis revealed significantly lower complexity (index NCI, i.e. nonlinear complexity index; \# indicates the statistically significant difference) in girls with major depression (D) compared to control group (C). While no differences in NCI were found in both supine positions (before and after orthostasis) these results stress the importance of cardiovascular variability testing during various standardized conditions (27)

\subsubsection{Multiscale entropy}

From recently developed nonlinear methods, measures of short-term complexity (various entropy measures) have shown to be promising for analysis of both heart rate and blood pressure signals $(13,26,28)$. However, since beat-to-beat RR interval and BP time series under healthy conditions have a complex temporal structure with correlations on multiple scales, single-scale based traditional entropy measures (e.g. Sample Entropy) may fail to account for the multiple time scales inherent in the complex physiological system dynamics. Therefore, a meaningful measure of complexity should take into account multiple time scales. Recently, Costa et al (2002) (29) introduced a new method to calculate entropy over multiple scales - Multiscale Entropy analysis (MSE).

$M S E$ is computed according to the procedure published by Costa (29). First step involves the construction of coarse-grained time series for each of the analyzed time scales determined by the scale factor $\tau$. In other words, coarse-grained time series for scale $\tau$ are obtained by taking arithmetic mean of $\tau$ neighbouring original values without overlapping. For scale 1, the coarse grained time series is simply the original time series. In the next step, SampEn values are calculated for each one of the coarse-grained time series and plotted against the scale factor. In this way, MSE describes the entropy (complexity) for various time scales of fluctuations within the analysed signal (Fig. 5). 


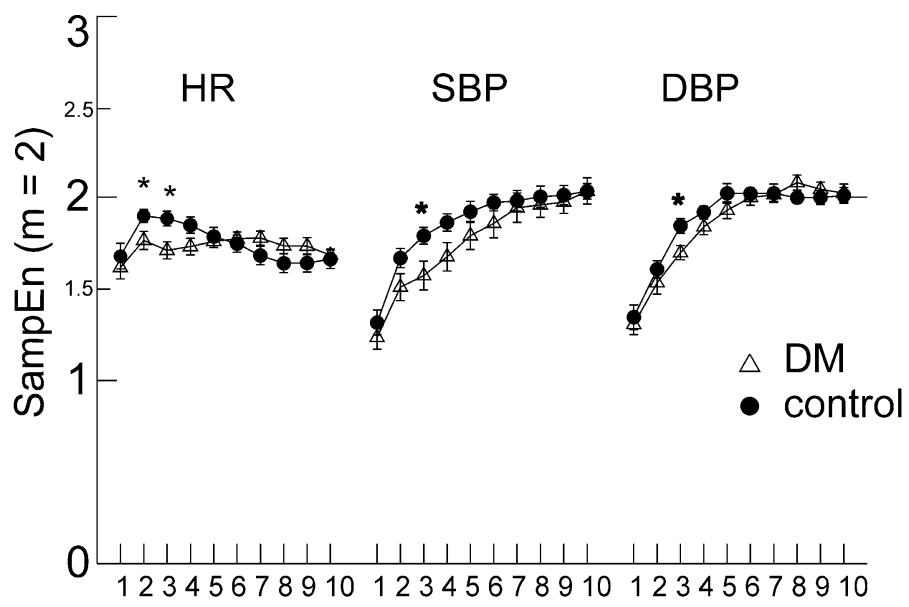

Fig. 5. Multiscale entropy analysis of heart rate (HR), systolic blood pressure (SBP) and diastolic blood pressure (DBP) oscillations during supine rest in patients with type 1 diabetes mellitus (open triangles, DM) and age and gender matched control subjects (full circles, control). In multiscale entropy analysis, Sample Entropy (SampEn) values are plotted against the corresponding time scale (in this study, for time scales 1-10). While reduction in complexity in DM was not detectable on scale 1 (original time series), significant loss of complexity in all three signals was detected on higher scales (2-3) indicating the importance of multiscale approach for cardiovascular signals complexity analysis. MSE was the only method capable to reveal subtle differences between both groups of subjects in blood pressure variability signals (17)

\subsection{Recurrences in cardiovascular dynamics}

Recurrence is a basic feature of many dynamical systems - it is defined as the repeated occurrence of a given state of the system in time. Recurrence plot (RP) is a graphical representation of such recurrences in a dynamical system (30) (Fig. 6).

\section{A: Supine}

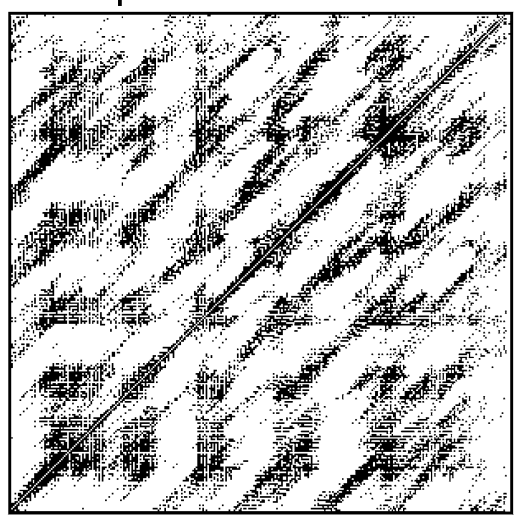

B: Standing

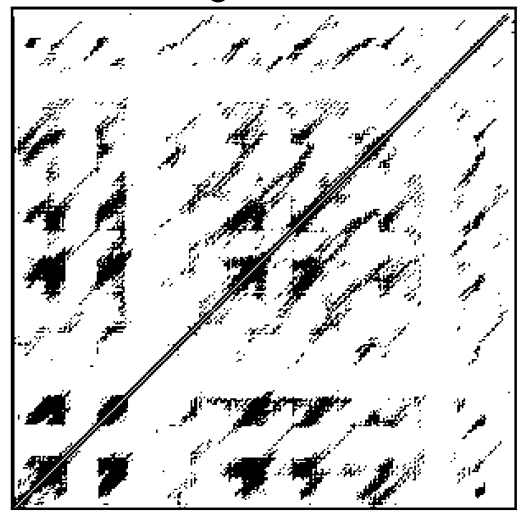

Fig. 6. A representative example of the recurrence plots (RP) constructed from heart rate oscillations during supine (A) and standing positions (B). RP was constructed as follows: any recurrence of state in time $i(x-$ axis) with the state in time $j(y$-axis) is plotted as a black dot. In both presented RPs, the percentage of recurrence (\%Rec; percentage of black points) was fixed to $5 \%$ - it means that the $5 \%$ of all points of RP were recurrent. The differences were found in the structure of patterns within RP: in standing position, higher proportion of points were grouped into diagonals (percentage of determinism - \%Det: $41 \%$ in supine vs. $78 \%$ in standing position) and verticals (Laminarity - Lam: 0.593 in supine vs. 0.860 in standing position) (31) 
As shown by analysis of model signals, RPs are sensitive to subtle qualitative changes in the system's dynamics (bifurcations) and could therefore be used for the characterization of shifts in basic cardiovascular parameters dynamics during both physiological and pathological conditions. The major advantage of recurrence plots is their applicability to short and nonstationary physiological time series. The power of RP also resides in its independence from constraining assumptions and limitations plaguing other analyses. Because recurrence structures are simply tallied within the signal, there is no need to pre-condition the data by filtering, linear detrending, or transforming the data to conform to any particular statistical distribution. For these reasons RPs has proven to be ideally suited for the study of numerous real-world systems (30).

The structures exhibited by RP reveal information on system properties and can be described quantitatively by recurrence quantification analysis $(R Q A)(32,33)$. RQA was useful for detection of heart rate dysregulation in various pathological conditions including chronic fatigue syndrome, hypertension, ventricular arrhythmia $(34,35)$.

\subsection{Time irreversibility}

Recently, it was shown that heart rate and blood pressure signals show a feature of time irreversibility. This phenomenon is specific for nonequilibrium systems with inherent complexity (36) and can be described as the lack of signal statistical properties stability after the signal time reversal. In other words, the signal is irreversible if its statistical properties are changed after the reversal of time (when one looks on the signal from right to left) (37). Several methods to quantify time asymmetry and their robustness and independence are introduced in this issue of Acta Medica Martiniana (38).

\section{Conclusion}

Autonomic function testing is essential to diagnose cardiovascular dysregulation which might result in fatal endpoints such as sudden cardiac death. The magnitude as well as nonlinear features of HRV may be easily quantified on relatively short recordings in a routine clinical setting, requiring only ECG and automated computer software, being neither cost nor labour intensive. HRV and BPV analysis is a useful tool for the diagnosis and monitoring of autonomic dysregulation and should include nonlinear analysis along with traditional time and frequency indices. Assessing HRV and BPV might also be suited to monitor different pharmacological and non-pharmacological treatment strategies.

While linear variability measures provide information on the overall magnitude of oscillations (quantitative analysis), nonlinear measures provide complementary information about qualitative features of the analyzed signal. Since analysis of variability and nonlinear characteristics (complexity, recurrences, time irreversibility, etc.) can provide independent information on cardiovascular system control, it is beneficial to use both groups of measures simultaneously in the studies demanding the application of HRV and BPV analysis as a noninvasive tool for the assessment of cardiovascular autonomic nervous system function. We suggest that simultaneous use of both groups of measures can increase the information value and thus improve the sensitivity and reliability of the detection and monitoring of the cardiovascular system dysregulation during various pathological conditions. To verify this, other studies on the HRV/BPV complexity measures application during specific pathological conditions are needed. 


\section{REFERENCES}

1. Takase B, Kitamura H, Noritake M, Nagase T, Kurita A, Ohsuzu F, Matsuoka T. Assessment of diabetic autonomic neuropathy using twenty-four hour spectral analysis of heart rate variability. A comparison with the findings of Ewing battery. Jpn Heart J 2002; 43: 127-135

2. Vinik AI, Maser RE, Mitchell BD, Freeman R. Diabetic Autonomic Neuropathy. Diabetes Care 2003; 26: 1553-1579

3. Javorka M, Javorkova J, Tonhajzerova I, Javorka K. Parasympathetic versus sympathetic control of the cardiovascular system in young patients with type 1 diabetes mellitus. Clin Physiol Funct Imaging 2005; 25: 270-274

4. Parati G, Mancia G, Di Rienzo M, Castiglioni P. Point: cardiovascular variability is/is not an index of autonomic control of circulation. J Appl Physiol 2006; 101: 676-8; discussion 681-2

5. Task Force of the European Society of Cardiology and the North American Society of Pacing and Electrophysiology. Heart rate variability. Standards of measurement, physiological interpretation, and clinical use. Eur Heart J 1996; 17: 354-81

6. Maser RE, Lenhard MJ. REVIEW: Cardiovascular autonomic neuropathy due to diabetes mellitus: Clinical manifestations, consequences, and treatment. J Clin Endocrinol Metab 2005; 90: 5896-5903

7. Laitinen T, Hartikainen J, Niskanen L, Geelen G, Länsimies E. Sympathovagal balance is major determinant of short-term blood pressure variability in healthy subjects. Am J Physiol 1999; 276: H1245-52

8. Eckberg DL. Physiological basis for human autonomic rhythms. Ann Med 2000; 32: 341-9

9. Aubert AE, Ramaekers D. Neurocardiology: the benefits of irregularity. The basics of methodology, physiology and current clinical applications. Acta Cardiol 1999; 54: 107-120

10. Beckers F, Verheyden B, Ramaekers D, Swynghedauw B, Aubert AE. Effects of autonomic blockade on non-linear cardiovascular variability indices in rats. Clin Exp Pharmacol Physiol 2006; 33: 431-439

11. Schreiber T. Interdisciplinary application of nonlinear time series methods. Phys Rep 1999; 308: 1-64

12. Raab C, Wessel N, Schirdewan A, Kurths J. Large-scale dimension densities for heart rate variability analysis. Phys Rev E 2006; 73: 041907

13. Baumert M, Baier V, Haueisen J, Wessel N, Meyerfeldt U, Schirdewan A, Voss A. Forecasting of life threating arrhythmias using the compression entropy of heart rate. Methods Inf Med 2004; 43: 202-206

14. Baumert M, Baier V, Schirdewan A, Truebner S, Voss A. Short- and long-term joint symbolic dynamics of heart rate and blood pressure indilated cardiomyopathy. IEEE Trans Biomed Eng 2005; 52: 2112-2115

15. Costa MD, Peng C-K, Goldberger AL. Multiscale analysis of heart rate dynamics: entropy and time irreversibility measures. Cardiovasc Eng 2008; 8: 88-93

16. Batchinsky AI, Cooke WH, Kuusela T, Cancio LC. Loss of complexity characterizes the heart rate response to experimental hemorrhagic shock in swine. Crit Care Med 2007; 35: 519-25

17. Trunkvalterova Z, Javorka M, Tonhajzerova I, Javorkova J, Lazarova Z, Javorka K, Baumert M. Reduced short-term complexity of heart rate and blood pressure dynamics in patients with diabetes mellitus type 1: multiscale entropy analysis. Physiol Meas 2008; 29: 817-828

18. Shannon CE. A mathematical theory of communication. The Bell System Technical J 1948; 27: 379-423, 623-656

19. Pincus SM, Gladstone IM, Ehrenkranz RA. A regularity statistic for medical data analysis. J Clin Monit. 1991;7: 335-45.

20. Richman JS, Moorman JR. Physiological time-series analysis using approximate entropy and sample entropy. Am J Physiol 20004; 278: H2039-49

21. Javorka M, Zila I, Balharek T, Javorka K. Heart rate recovery after exercise: relations to heart rate variability and complexity. Braz J Med Biol Res 2002; 35: 991-1000

22. Ziv J, Lempel A. A universal algorithm for sequential data compression. IEEE Trans Inf Theory 1977; 23: 337-343

23. Javorka M, Trunkvalterova Z, Tonhajzerova I, Javorkova J, Javorka K, Baumert M. Short-term heart rate complexity is reduced in patients with type 1 diabetes mellitus. Clin Neurophysiol 2008; 119: 1071-1081

24. Voss A, Kurths J, Kleiner HJ, Witt A, Wessel N, Saparin P, Osterziel KJ, Schurath R, Dietz R. The application of methods of non-linear dynamics for the improved and predictive recognition of patients threatened by sudden cardiac death. Cardiovasc Res 1996; 31: 419-433

25. Porta A, Guzzetti S, Montano N, Furlan R, Pagani M, Malliani A, Cerutti S. Entropy, entropy rate, and pattern classification as tools to typify complexity in short heart period variability series. IEEE Trans Biomed Eng 2001; 48: 1282-91. 
26. Porta A, Faes L, Mase M, D`Addio G, Pinna G D, Maestri R, Montano N, Furlan R, Guzzetti S, Nollo G, Malliani A. An integrated approach based on uniform quantization for the evaluation of complexity of short-term heart period variability: Application to 24h Holter recordings in healthy and heart failure humans. Chaos 2007; 17: 015117

27. Tonhajzerova I, Ondrejka I, Javorka K, Turianikova Z, Farsky I, Javorka M. Cardiac autonomic regulation is impaired in girls with major depression. Prog Neuropsychopharmacol Biol Psychiatry 2010; 34: 613-618

28. Wessel N, Bauernschmitt R, Wernicke D, Kurths J, Malberg H. Autonomic cardiac control in animal models of cardiovascular diseases. I. Methods of variability analysis. Biomed Tech (Berl) 2007; 52: 43-9

29. Costa M, Goldberger AL, Peng CK. Multiscale entropy analysis of complex physiologic time series. Phys Rev Lett 2002; 89: 068102

30. Marwan N, Romano MC, Thiel M, Kurths J. Recurrence plots for the analysis of complex systems. Phys Rep 2007; 438: 237-329

31. Javorka M, Turianikova Z, Tonhajzerova Z, Javorka K, Baumert M. The effect of orthostasis on recurrence quantification analysis of heart rate and blood pressure dynamics. Physiol Meas 2009; 30: 29-41

32. Webber CL, Zbilut JP. Dynamical assessment of physiological systems and states using recurrence plot strategies. J Appl Physiol 1994; 76: 965-973

33. Marwan N, Wessel N, Meyerfeldt A, Schirdewan A, Kurths J. Recurrence plot based measures of complexity and its application to heart rate variability data. Phys Rev E 2002; 66: 026702

34. Wessel N, Marwan N, Meyerfeldt U, Schirdewan A, Kurths J. Recurrence quantification analysis to characterise the heart rate variability before the onset of ventricular tachycardia. Lect Notes Comput Sc 2001; 2199: 295-301

35. Naschitz JE, Itzhak R, Shaviv N, Khorshidi I, Sundick S, Isseroff H, Fields M, Priselac M, Yeshurun D, Sabo E. Assessment of cardiovascular reactivity by fractal and recurrence quantification analysis of heart rate and pulse transit time. J Hum Hypertens 2003; 17: 111-118

36. Hou F, Zhuang J, Bian C, Tong T, Chen Y, Yin J, Qiu X, Ning X. Analysis of heartbeat asymmetry based on multi-scale time irreversibility test. Physica A 2010; 389: 754-760

37. Porta A, Casali KR, Casali AG, Gnecchi-Ruscone T, Tobaldini E, Montano N, Lange S, Geue D, Cysarz D, Van Leeuwen P. Temporal asymmetries of short-term heart period variability are linked to autonomic regulation. Am J Physiol 2008; 295: R550-557

38. Chladekova L, Turianikova Z, Tonhajzerova I, Calkovska A, Javorka M. Time irreversibility of heart rate oscillations during orthostasis. Acta Med Mart 2011; (Suppl. 1):39-44

\section{Acknowledgement}

This paper was supported by project of Center of Excellence for Perinatological Research No 26220120016, co-financed from EU sources.

Received: December, 21, 2010

Accepted: February, 6, 2011 